DE RGUASCALIENTES

\title{
Análisis prospectivo del uso de energía solar: Caso Colombia
}

\author{
Prospective analysis for using solar energy: Colombia's case
}

\begin{abstract}
$\gamma^{2}$
María Fernanda Serrano-Guzmán*凶, Diego Darío Pérez-Ruiz*, John Freddy Galvis-Martínez**, Marlon Leonardo Rodríguez Sierra**, Sandra Natalia Correa Torres****

Serrano-Guzmán, M. F., Pérez-Ruiz, D. D., Galvis-Martínez, J. F., Rodríguez Sierra, M. L., \& Correa Torres, S. N. (2017). Análisis prospectivo del uso de energía solar: Caso Colombia. Investigación y Ciencia de la Universidad Autónoma de Aguascalientes, 25 (71), 85-93.
\end{abstract}

RESUMEN

La preocupación por el aumento en las emisiones de gases efecto invernadero ha impulsado el abastecimiento energético a partir de fuentes como la energía solar. Sin embargo, la puesta en marcha de una central solar genera impactos adversos sobre el ambiente. Este artículo, derivado de la investigación sobre fuentes energéticas no convencionales, se centra en la identificación de los impactos en las fases de operación y puesta en marcha de centrales fotovoltaica y térmica y presenta además una prospectiva de las emisiones esperadas de $\mathrm{CO}_{2}$ si se opta por este energético en un país como Colombia. El análisis prospectivo se realizó con LEAP empleando los valores de emisiones de $\mathrm{CO}_{2}$ encontrados. En este estudio se evidencia que aunque existen impactos positivos, la presencia de impactos negativos exige la implementación de estrategias de mitigación ambiental de los daños causados. Se concluyó que es viable el uso de la energía solar.

Palabras clave: recurso natural; estación de potencia; energía solar; dióxido de carbono; impacto; emisiones.

Keywords: natural resource; power station; solar energy; carbon dioxide; impact; emissions.

\section{Recibido: 13 de octubre de 2015, aceptado: 19 de agosto de 2016}

* Ingeniería Civil, Pontificia Universidad Javeriana de Cali. Cl. 18 \#118250, C. P. 760031, Cali, Valle del Cauca, Colombia. Correo electrónico: maria.serrano@javerianacali.edu.co; ddperez@javerianacali.edu.co

** Ingeniería Civil, Universidad Pontificia Bolivariana Bucaramanga. Autopista Piedecuesta kilómetro 7, C. P. 681007, Bucaramanga, Santander, Colombia. Correo electrónico: john.galvism@upb.edu.co; leonardomarlon7@gmail.com

*** Ingeniería Ambiental, Universidad Pontificia Bolivariana Bucaramanga. Autopista Piedecuesta kilómetro 7, C. P. 681007, Bucaramanga, Santander, Colombia. Correo electrónico: sandra.correa@upb.edu.co

$\triangle$ Autor para correspondencia
ABSTRACT

There is a whole concern regarding the increasing in greenhouse gas emissions; due to this, it is encouraging the use of non-conventional energy sources as solar energy. Nevertheless, the implementation of solar power plants generates impacts on the environment. This article focuses on identifying impacts during the operation and commissioning of photovoltaic and thermal power plants and shows a prospective of the expected emissions of $\mathrm{CO}_{2}$ if this source is applied in a country like Colombia. The prospective analysis was done using LEAP that includes $\mathrm{CO}_{2}$ emissions values that were found. This study shows that although there are positive impacts, the presence of negative impacts requires the implementation of mitigation strategies for environmental damage. This study concluded that the use of solar energy is feasible.

\section{INTRODUCCIÓN}

El desarrollo industrial en conjunto con el crecimiento poblacional está aumentando el consumo de energía suministrada a través de hidrocarburos, centrales hidroeléctricas y en algunos casos requiere energías no convencionales (Hernandez et al., 2014), todo esto con los costos económicos, sociales y ambientales respectivos. Justamente, debido a que se comprometen los recursos físicos y bióticos disponibles, en ocasiones de manera no sustentable y no sostenible, existe una fuerte tendencia al uso de energías no convencionales, las cuales liberan menos contaminantes responsables del detrimento en la calidad del ambiente.

La motivación del uso de la energía solar se fundamenta en la proyección que la Unidad de Planeación Minero Energética (UPME) (2015) hizo 
IIVESTIGACIÓn Y CIERCIA DE LA UNIVERSIDAD AUTÓNOMA

con relación a esta fuente energética de la cual se proyecta que para 2028 alrededor de $13.75 \%$ de los 3,275 MW instalados corresponda a la misma (UPME, 2015), pero a pesar de los beneficios que representa, existen impactos ambientales que deben ser mitigados cuando un país opta por este sistema energético.

El presente artículo señala los impactos ambientales generados durante su producción y uso. Asimismo, se incluye un análisis prospectivo aplicado para el escenario de Colombia, que demuestra la conveniencia de su aplicación atendiendo a las bajas emisiones atmosféricas.

\section{Identificación de impactos ambientales}

La producción y uso de energía solar repercute de manera positiva y negativa en el ambiente. La primera se hace evidente porque este energético produce hasta $18.8 \%$ menos emisiones de $\mathrm{CO}_{2}$ (Hernandez et al., 2014), favorece el proceso incluyente de energía para poblaciones rurales y se hace parte de la oferta energética de las comunidades con posibilidad de oportunidades laborales para proveedores de celdas y paneles y para los responsables del funcionamiento de la planta de producción, que incluso puede estar ubicada en terrenos degradados pero que se recuperan con la construcción de la central solar. Todas estas justificaciones positivas hacen que este energético sea un medio para la mejora en la calidad de vida de los países en desarrollo (Burney, Woltering, Burke, Naylor, \& Pasternak, 2010; Tsoutsos, Frantzeskaki, \& Gekas, 2005). Con respecto a los segundos, se asocian más a la fase de conservación y desmantelamiento de la planta por la permanencia que puedan tener en el paisaje las adecuaciones realizadas durante la operación de la central solar, cuya vida útil puede ser de 25 a 40 años (UPME, 2015).

Además de lo señalado anteriormente, un sistema de este tipo de combustible puede afectar la biodiversidad, el recurso hídrico, suelo y cobertura vegetal, la salud humana y la calidad del aire. Es un hecho que los impactos serán evidenciados en las distintas etapas del ciclo de vida de la central solar y tendrán magnitudes diferenciales (UPME, 2015).

Las grandes centrales solares requieren grandes extensiones de tierra para la instalación de celdas o paneles solares y éstos alteran el paisaje, y pueden causar desertificación (Bastida, Moreno, Hernández, \& García, 2006), generan barreras para la movilidad de las especies y en algunos casos obligan a la migración definitiva de las mismas afectando sensiblemente a la biodiversidad (UPME, 2015). Por otro lado, la remoción de la cobertura vegetal hace que se favorezca la liberación de carbono a la atmósfera en forma de dióxido de carbono $\left(\mathrm{CO}_{2}\right)$, lo que disminuye la concentración de carbono en el recurso suelo (UPME, 2015) esto atenta contra la calidad del suelo, afecta la capacidad para la sustentabilidad de los sistemas productivos y repercute en la fertilidad y la productividad de la tierra para la producción de alimentos y para la seguridad alimentaria (Fthenakis, \& Chul Kim, 2009). También la instalación de paneles solares, que pueden elaborarse con plomo, níquel, arsénico y en ocasiones con teluro de cadmio, sustancias que requieren adecuados procesos de disposición (IPCC, 2012; Sinha et al., 2008) en consideración a su toxicidad (IEA, 2014).

Finalmente, durante la operación de la central solar es común llevar a cabo actividades de lavado de paneles o espejos, con lo cual se puede contaminar el agua. Para esta labor se ha encontrado que son necesarios alrededor de $0.02 \mathrm{~m}^{3}$ de agua por megavatio hora de energía (UPME, 2015). En este caso, aunque el impacto por consumo de agua es bajo, la disposición final de las aguas con sedimentos, cuando son descargadas al suelo durante el lavado, afectan la cobertura vegetal, lo que propicia espacios para la erosión. A lo anterior se suma la ocupación permanente de la tierra por los paneles y las redes de transmisión, así como la afectación sobre la visibilidad tanto para los vehículos que ocupan las vías perimetrales como las aves que ocupan el espacio aéreo (Hernandez et al., 2014).

A continuación se resumen los impactos más relevantes en los distintos recursos naturales:

Aire. Son similares a los que se producen por cualquier instalación industrial de gran escala y que, por tanto, pueden afectar la calidad del aire, la salud de los empleados de la planta y la de los residentes en las inmediaciones de la central solar (Office of Indian Energy and Economic Development, 2015). Inclusive, por acción del viento, pueden llegar a liberarse patógenos presentes en el suelo que pueden movilizarse a través de las corrientes de aire (Pepper, Gerba, \& Newby, 2009). Además, ante una eventual movilidad de material particulado durante la operación de la planta, se producen cortinas de polvo que disminuyen la visibilidad en las carreteras y pueden llegar a afectar los cuerpos de agua (Ravi et al., 2011). 
Durante la fase de clausura, la inadecuada disposición de las celdas fotovoltaicas que contienen cadmio, arsénico y sílice, puede favorecer la liberación de estos compuestos, exponiendo a las especies vegetales, animales y al hombre a los efectos nocivos por el contacto. Por otro lado, para contrarrestar estos efectos se emplean productos químicos de materiales supresores de polvo, líquidos refrigerantes y fluidos de transferencia de calor, y las sustancias que contienen pueden contaminar la superficie del agua subterránea y los reservorios de aguas profundas (Fthenakis, \& Chul Kim, 2009; Gagnon, Bélanger, \& Uchiyama, 2002; Tsoutsos et al., 2005).

Biota animal. El auge en las fuentes de energía solar está motivando la exportación de este tipo de energía y ha alcanzado fronteras internacionales (Deluchi, \& Jacobson, 2011). Como ejemplo de esto, actualmente se está desarrollando un programa para transmitir energía de Oriente Medio y las regiones del norte de África a los países europeos, para lo cual se contempla la construcción de más de 78,000 km de líneas de transmisión para el año 2050 (Trieb, Schillings, Pregger, \& O'Sullivan, 2012). Con seguridad, la construcción de este tipo de redes extensas de líneas de transmisión impacta a corto y largo plazo las especies del entorno en donde se desarrolla el trazado en lo concerniente al desplazamiento de la fauna silvestre, eliminación de la cubierta vegetal $y$, en general, a la degradación de la calidad del hábitat (Trieb et al., 2012).

La energía solar y su relación con las emisiones de $\mathrm{CO}_{2}$ El cambio climático está ocurriendo por la presencia de los gases de efecto invernadero (GEI), derivada de las actividades humanas que alteran la composición química, así como por la variabilidad climática atribuible a causas naturales (IPCC, 2014), lo que ha producido un aumento promedio aproximado de un grado Celsius en el último siglo, una elevación de aproximadamente $17 \mathrm{~cm}$ en el nivel del mar, pérdida de la biodiversidad marina, reducción de la captura de dióxido de carbono y disminución de la cobertura de hielo en los casquetes polares; efectos que repercuten en el hábitat de los asentamientos humanos y de los animales y vegetales (Herrán, 2012).

El dióxido de carbono es uno de los gases traza más comunes e importantes en el sistema atmósfera-
océano-Tierra, es el más importante GEl asociado a actividades humanas y el segundo gas más importante en el calentamiento global después del vapor de agua (Benavides Ballesteros, \& León Aristizábal, 2007). Este gas se libera por fuentes antropogénicas y naturales. En relación con las actividades humanas el $\mathrm{CO}_{2}$ se emite, principalmente, por el consumo de combustibles fósiles (carbón, petróleo, sus derivados y gas natural) y leña para generar energía, por la tala y quema de bosques. Por otro lado, en el ciclo natural del carbono, el $\mathrm{CO}_{2}$ cumple un rol principal en gran número de procesos biológicos. Las mayores concentraciones de $\mathrm{CO}_{2}$ se presentan en el hemisferio norte, donde se encuentra la totalidad de los países industrializados responsables exclusivos de este aumento en la mayoría de los GEl en la atmósfera desde la revolución industrial, a mediados del siglo XIX (Benavides Ballesteros, \& León Aristizábal, 2007).

El $\mathrm{CO}_{2}$ es el GEl que más ha contribuido en el forzamiento radiativo que aumentó $20 \%$ desde 1995 hasta 2005, el cambio más grande observado o inferido para una década en los últimos 200 años. El forzamiento radiativo para el $\mathrm{CO}_{2}$ corresponde a $1.66 \mathrm{~W} / \mathrm{m}^{2}$, frente a $0.48 \mathrm{~W} / \mathrm{m}^{2}$ aportado por el $\mathrm{CH}_{4}, 0.35 \mathrm{~W} / \mathrm{m}^{2}$ por el ozono troposférico y $0,16 \mathrm{~W} /$ $\mathrm{m}^{2}$ por el $\mathrm{N}_{2} \mathrm{O}$ y algunos halocarbonos (Benavides Ballesteros, \& León Aristizábal, 2007). Como dato significativo, cabe resaltar que países como China y Estados Unidos se ubicaron en el primer y segundo lugar, respectivamente, como mayores productores de emisiones de $\mathrm{CO}_{2}$, seguidos por India, Rusia y Japón, que ocuparon el tercer, cuarto y quinto lugar, respectivamente.

La problemática generada por la sobreproducción de $\mathrm{CO}_{2}$ a nivel mundial representa una preocupación a nivel mundial, en donde países como China y Estados Unidos han ocupado el primer y segundo lugar en producción del mismo (Marland, Boden, \& Andres, 2013). Los inconvenientes ambientales por las energías convencionales han motivado el uso de otras fuentes de energía, es entonces cuando surge la solar como un sistema con ventajas medioambientales evidentes en comparación con las fuentes convencionales. Su principal ventaja está relacionada con las bajas emisiones de $\mathrm{CO}_{2}$ (Tsoutsos et al., 2005) frente a otras no convencionales (tabla 1). 
Tabla 1

Emisiones de $\mathrm{CO}_{2}$ por diferentes tecnologías energéticas

\begin{tabular}{ccccc}
\hline Emisión & $\begin{array}{c}\text { Central térmica } \\
\text { solar }\left(^{* 1}\right)\end{array}$ & $\begin{array}{c}\text { Central de generación de energía } \\
\text { fotovoltaica }\left(^{* 1}\right)\end{array}$ & ${\text { Gas }{ }^{* 2}}^{\text {Petróleo }{ }^{* 2}}$ & ${\text { Carbón }{ }^{* 2}}^{*}$ \\
\hline $\mathrm{CO}_{2}(\mathrm{Kg} / \mathrm{KWh})$ & 1.4 & $0.6-1.0$ & 181.08 & 253.83 \\
\hline Nota: Adaptada de ${ }^{* 1}$ Tsoutsos et al. (2005) y ${ }^{* 2}$ Naturalgas.org (2013). & &
\end{tabular}

En producción de silicio para celdas solares en concentraciones de 1 p.p.m., se requiere un consumo adicional de energía de 15 a $20 \mathrm{kWh} / \mathrm{kg}$ de silicio monocristalino y de $50 \mathrm{kWh} / \mathrm{kg}$ de silicio policristalino (Dubey, Jadhav, \& Zahirova, 2013). Asimismo, para la producción de baterías se emplean diferentes metales pesados, los cuales aportan a la huella en el agua producida durante la elaboración de estas baterías y ello conlleva a la liberación de $\mathrm{CO}_{2}$ según el metal que se emplee (Sullivan, \& Gaines, 2012) (tabla 2).

Tabla 2

Emisiones y contaminación en el agua por kg de batería producida

\begin{tabular}{ll}
\hline \multicolumn{1}{c}{ Tecnología } & $\mathrm{CO}_{2}(\mathrm{~g} / \mathrm{kg})$ \\
\hline Níquel-hidruro metálico $(\mathrm{NiMH})$ & 13.6 \\
\hline Plomo-ácido $(\mathrm{PbA})$ & 3.2 \\
\hline Niquel-cadmio $(\mathrm{NiCd})$ & 9.6 \\
\hline Sodio-azufre $(\mathrm{Na} / \mathrm{S})$ & 14.3 \\
\hline Total & 40.7 \\
\hline
\end{tabular}

Nota: Adaptada de Sullivan y Gaines (2012).

El auge de la energía solar se ha elevado tanto en el uso de la tecnología de celdas fotovoltaicas como en la energía solar concentrada (Torresol Energy Investments, 2010 a). Las plantas solares térmicas con colectores de cilindros parabólicos se componen de lazos o hileras de espejos de forma parabólica que concentran la radiación solar en un tubo colector central por el que circula un aceite térmico que se calienta para alcanzar una temperatura cercana a los $400^{\circ} \mathrm{C}$. Este aceite caliente se traslada a un intercambiador térmico, donde se vaporiza el agua que acciona un generador que, a su vez, inyecta la energía eléctrica a la red en una turbina de vapor (Torresol Energy Investments, 2010 b).

Para la producción de una planta que se desarrolla en un área de $2 \mathrm{~km}^{2}$ y tiene la capacidad de producir $50 \mathrm{MW}$ se consumen 97,691 MWh/año de gas natural y 16,338 MWh/año de electricidad convencional; además, se generan 187,581 MWh/h de energía (Burkhardt, Heath, \& Turchi, 201 1), proceso en el cual se liberan de 0.20 a $0.34 \mathrm{~g} / \mathrm{KWh}$ de $\mathrm{SO}_{2}$ y 0.18 a $0.30 \mathrm{~g} / \mathrm{kWh}$ de NOx (Gutiérrez, 2001). Con relación a la huella en el agua, estas plantas consumen cantidades diferentes en las distintas etapas del ciclo de vida (tabla 3).

En las plantas de torre central, los heliostatos (espejos planos) reflejan la radiación solar en un receptor situado en lo alto de la torre por la cual circulan sales de nitrato fundidas. Dichas sales son impulsadas desde un "tanque frío" hasta el receptor situado en la parte de arriba, donde se calientan hasta alcanzar $565^{\circ} \mathrm{C}$. Las sales calientes bajan al intercambiador de calor para generar vapor de agua (US Department of Energy, 2008) y libera de esta forma de 20 a $50 \mathrm{~g} / \mathrm{kWh}$ de $\mathrm{CO}_{2}$ (Nelson, Ghambir, \& Ekins-Daukes, 2014), de 0.13 a $0.27 \mathrm{~g} / \mathrm{kWh}$ de $\mathrm{SO}_{2}$ y de 0.06 a 0.13 de NOx g/kWh (Gutiérrez, 2001).

Tabla 3

Emisiones y consumo de agua de los colectores de cilindro-parabólicos

\begin{tabular}{lll}
\hline \multicolumn{1}{c}{ Fase del ciclo de vida } & \multicolumn{1}{c}{ Emisiones $\left(\mathrm{gCO}_{2} \mathrm{eq} / \mathrm{kWh}\right)$} & Agua (litros $/ \mathrm{kWh})$ \\
\hline Fabricación & 13 & 0.50 \\
\hline Construcción & 1.8 & 0.033 \\
\hline Operación & 11 & 0.55 \\
\hline Desmantelamiento & 0.12 & 0.00053 \\
\hline Disposición & 2.1 & 0.0074 \\
\hline Total & 28 & 1.1 \\
\hline
\end{tabular}

Nota: Adaptada de Torresol Energy Investments (2010 a). Elaboración propia. 


\section{InVESTIGACIÓn Y CIERCIA DE LA UחIVERSIDAD AUTÓnOMA DE RGUASCALIEחTES}

\section{Políticas relevantes hacia el uso de energía solar como alternativa para la mitigación del cambio climático}

A pesar del impacto negativo y considerando los beneficios por la baja producción de $\mathrm{CO}_{2^{\prime}}$ la energía solar se considera una alternativa energética limpia que produce menor cantidad de emisiones contaminantes que los tipos de energía convencionales. Por esto, los países desarrollados trabajan alrededor de un programa de transición mediante políticas que incentivan el uso de energías no convencionales. A continuación, se presenta una síntesis de las iniciativas más sobresalientes:

- En Estados Unidos se estimó que por lo menos 2\% de la electricidad sería suministrada por sistemas solares fotovoltaicos, mismos que efectivamente suministraron 50, $000 \mathrm{MW}$ en instalaciones solares fotovoltaicas, así como más de 6, $600 \mathrm{MW}$ de energía solar concentrada (US Department of Energy, 2008). Para el periodo comprendido entre 2009 y 2010 se evidenció un incremento de $11 \%$ en el uso de energías renovables y $77 \%$ en el uso de energía solar; en 2012 alcanzó la máxima capacidad acumulada de energía solar fotovoltaica de 7, $200 \mathrm{MW}$, empleada en diferentes Estados (Sahu, 2015).

- En China, debido al crecimiento industrial y a los asentamientos humanos, ha aumentado el consumo de energía (Martinot, 2001) y ha desencadenado problemas de contaminación ambiental, con hasta $75 \%$ por contaminación derivada del consumo de carbón empleado para generación eléctrica (Yanrui, 2003). Por lo anterior, como estrategia gubernamental fundamentada en los incentivos y directrices de gestión de nuevas fuentes de energía (National Renewable Energy Laboratory, 2004), siguiendo en este esquema político, el gobierno chino espera reducir la emisión de dióxido de carbono en 17\% para 2020 (Sahu, 2015).

- En Alemania, luego de la comisión de cambio climático de 2004, en donde se estableció la necesidad mundial de reducir el dióxido de carbono y gas metano en $80 \%$ para 2050 , se elaboró el Plan de Acción Nacional de Energías Renovables (PANER) (Lauber y Mez, 2004). Por ello se proyectó el uso de la energía renovable en $38 \%$ para $2020,50 \%$ para 2030, 65\% para 2040 y $80 \%$ para 2050, con una capacidad total acumulada de energía solar por fotoceldas de 51,750 MW7 adicionados de 3,500 MW por año. Conviene destacar también que Alemania es uno de los países que mayor número de investigaciones ha realizado sobre uso de energía solar y llegó a registrar en 2010 un total de 290 patentes relacionadas (Grigoleit, \& Lenkeit, 2012).

- En cuanto a Japón, el gobierno estableció un programa incremental de uso de energía renovable para enfrentar la alta demanda de energía, de modo que para 2030 se proyecta que entre $25 \%$ y $35 \%$ de la generación total sea de tipo renovable, invirtiendo 700 millones de dólares en energía nueva y renovable. Según la Agencia de Recursos Naturales y Energía (ANRE), las energías renovables alcanzaron 1, 662, 000 $\mathrm{kW}$, de los cuales $93.8 \%$ correspondió a energías fotovoltaicas (Ravi et al., 2011). Para 2050, según el Instituto de Políticas de Energía Sostenible (ISEP), se espera la implementación de $100 \%$ de consumo de energía mediante abastecimiento por modos no convencionales (Fthenakis, Moskowitz, \& Lee, 1984).

\section{Proyección del uso de energía solar para el caso de Colombia}

Con relación a Colombia, la ley 1715 de 2014 señala que se debe buscar el aprovechamiento del recurso solaren proyectos de urbanización municipal o distrital, en edificaciones oficiales, en los sectores industrial, residencial y comercial planteando incentivos tributarios para los constructores que implementen fuentes de energía limpias en sus proyectos. Aun así, el desarrollo de este tipo de energía en Colombia está en proceso de implementación.

Para la proyección del tipo de energía estudiado se propone el imaginario de materias prima y energía que requiere una planta torre central como la existente en España, país que tiene una población cercana a la de Colombia. Se consideró el funcionamiento de la central por un lapso de 6,230 h/ año y una eficiencia campo solar de $45.6 \%$ (Tsoutsos et al., 2005).

En el escenario de autoabastecimiento energético se espera en el mediano plazo una mayor eficiencia en los procesos agrícolas e industriales, y según lo mismo que se tome la decisión de implementar la energía solar (por lo menos en $0.6 \%$ de la oferta energética) (UPME, 2015). Se presenta una perspectiva de la penetración de este tipo de energía (tabla 4). 
Tabla 4

Escenario autosuficiencia

\begin{tabular}{|c|c|c|c|c|c|}
\hline & 2010 & 2020 & 2030 & 2040 & 2050 \\
\hline Solar (fotovoltaica) & $0 \%$ & $1 \%$ & $2 \%$ & $2.5 \%$ & $3 \%$ \\
\hline Termosolar & $0 \%$ & $0 \%$ & $1 \%$ & $1.5 \%$ & $2 \%$ \\
\hline
\end{tabular}

Nota: Fuente: Rodríguez y Serrano-Guzmán (2015).

Tabla 5

Emisiones de $\mathrm{CO}_{2}$

\begin{tabular}{ccc}
\hline Emisión & Central térmica solar & $\begin{array}{c}\text { Central de generación de } \\
\text { energía fotovoltaica }\end{array}$ \\
\hline $\mathrm{CO}_{2}(\mathrm{~kg} / \mathrm{kWh})$ & 1.4 & $0.6-1.0$ \\
\hline $\mathrm{CO}_{2}\left(\mathrm{~kg} / \mathrm{m}^{2}\right)$ & 840 & $---\mathbf{- -}^{--}$ \\
\hline
\end{tabular}

Nota: Adaptada de Tsoutsos et al. (2005).

Para la proyección de las dos tecnologías (fotovoltaica y termosolar) en 2050, se consideraron los valores de emisiones de $\mathrm{CO}_{2}$ reportados en la literatura y con la ayuda de la herramienta computacional LEAP (Long range Energy Alternatives Planning System), se proyectaron según la demanda de la tabla 4 los valores consignados en la tabla 5.

Según los resultados arrojados por dicho software, se espera que para 2050 las emisiones de $\mathrm{CO}_{2}$ provenientes de esta planta solar (fotovoltaica) sean de 0.3 millones de toneladas de $\mathrm{CO}_{2}$ frente a 0.025 de 2020. En el caso de la proyección de emisiones de $\mathrm{CO}_{2}$ por el uso de energía solar térmica (termosolar) se esperan alrededor de 350, 000 t de $\mathrm{CO}_{2}$ para el año 2050, frente a 40, 000 t hacia 2025 (figura 1). La comparación entre las dos alternativas deja entrever que la energía termosolar produce mayor cantidad de emisiones que la solar fotovoltaica. El incremento en las emisiones de $\mathrm{CO}_{2}$ en la primera ocurre porque se requiere mayor cantidad de agua para la producción de energía debido a sus procesos térmicos, y aunque esta tecnología se proyecta para aparecer 10 años después de la fotovoltaica, genera mayores emisiones de $\mathrm{CO}_{2}$ que ésta llegando al año 2050.

\section{DISCUSIÓN}

Existe una preocupación mundial por el deterioro del ambiente y particularmente por el aumento en la producción de GEI. La solar se perfila como una fuente energética limpia que se caracteriza por bajas emisiones. Aun así, se generan impactos negativos derivados de la etapa de producción de paneles y del desmantelamiento de las centrales. La connotación de residuos electrónicos de estas

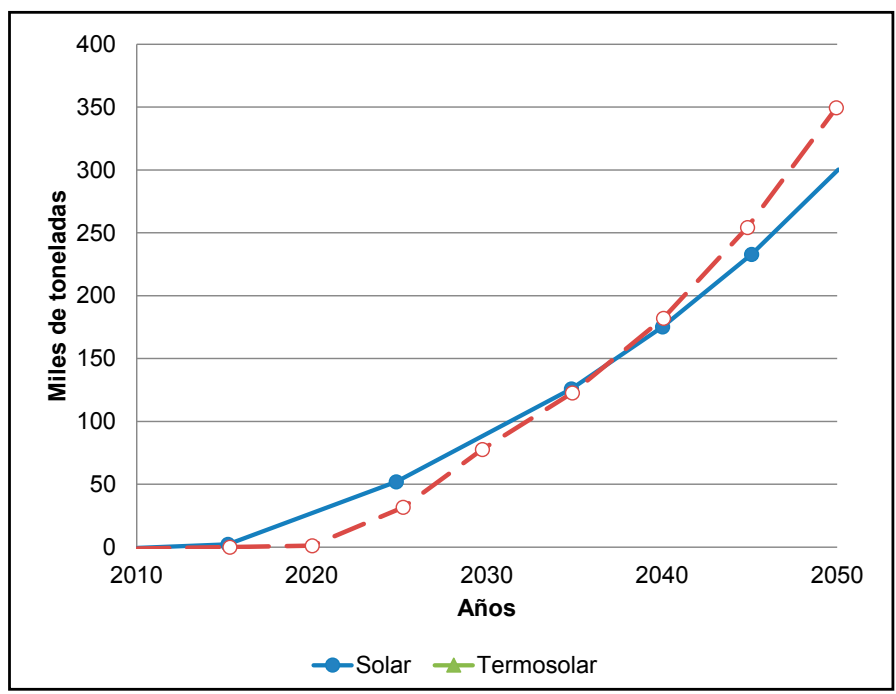

Figura 1. Comparación de las emisiones de $\mathrm{CO}_{2}$ de energía solar (fotovoltaica) y energía termosolar.

Fuente: Rodríguez y Serrano-Guzmán (2015).

centrales obliga a que se establezca un programa de disposición de desechos adecuado.

La recomendación para los países en vías de desarrollo es diseñar un programa de incorporación de la energía solar de forma gradual, incremental; de la misma forma, Fthenakis et al. (1984) describe la forma como Japón diseñó su estrategia de inclusión en el mercado. Igualmente, es necesario que se propongan incentivos tributarios, tanto para el constructor que en sus sistemas de abastecimiento incluya energías no convencionales, por ejemplo, para el funcionamiento de ascensores e iluminación de áreas comunes, como para las comunidades que adopten este sistema. Resulta conveniente que en cada región se realice la modelación correspondien- 


\section{IIVESTIGAGIÓn Y CUERCIA DE LA UחIVERSIDAD AUTÓNOMA DE RGUASCHLIETTES}

te de los energéticos no convencionales que se espera implementar porque las tecnologías disponibles varían entre las regiones y se debe seleccionar la que es viable de conseguir en el mercado. Es importante destacar también que la posibilidad de llevar servicio eléctrico a comunidades no interconectadas a la red central se viabiliza con la implementación de un sistema solar.

Para que este tipo de iniciativa sea ambientalmente aceptada se deben acometer medidas de control y mitigación de las actividades que producen efectos adversos. Se plantean las siguientes acciones preventivas y mitigadoras:

- Las afectaciones al recurso hídrico se pueden controlar mediante la construcción de trampas de sedimentos y canales perimetrales; en las primeras se recogen los residuos que por el arrastre han alcanzado los cuerpos de agua. En los segundos, mediante la rugosidad y pendiente del canal se controla la movilidad del material que puede desplazarse por acción de la escorrentía o del viento.

- El control de drenaje superficial, además de mitigar la cantidad de sólidos suspendidos en el agua, favorece las repercusiones de la escorrentía superficial sobre la cobertura vegetal. Se recomienda la siembra de árboles y especies de la región en la periferia de la central solar. No se recomienda la reposición de la biomasa con especies de gran altura, a menos que las mismas se ubiquen fuera del área de influencia de la central porque pueden producir sombra.
- Por otro lado, el movimiento de material particulado se puede controlar mediante la siembra de barreras vivas perimetrales y el uso de nanomateriales fijadores de este material.

- En cuanto a la biodiversidad, además del cálculo de la biomasa equivalente, es útil que se recreen escenarios de hábitat en las inmediaciones de las estaciones solares.

CONCLUSIONES

La energía solar es parte de la oferta energética de varios países. En aquellos en donde existen políticas definidas con inversión en tecnología se han logrado proyectos solares eficientes y los impactos ambientales generados durante la operación de las centrales solares pueden ser mitigados con mantenimiento preventivo a la infraestructura existente en las inmediaciones de la estación solar. Asimismo, cuando se requiera mantenimiento correctivo debe existir un programa ambiental para la disposición de los residuos líquidos o sólidos que se generan.

La alternativa de energía solar puede ser una opción viable para abastecimiento en comunidades rurales. Además, aunque el uso de la tecnología termosolar requiere mayor cantidad de agua que la fotovoltaica debido a sus procesos térmicos, ambas liberan menos $\mathrm{CO}_{2}$ que los combustibles fósiles. La decisión de la puesta en marcha de uno u otro sistema requiere el apoyo de entidades gubernamentales mediante incentivos tributarios a los productores y consumidores de energía solar.

REFERENCIAS

- Bastida, F., Moreno, J., Hernández, T., \& García, C. (2006). Microbiological activity in a soil 15 years after its devegetation. Soil Biology and Biochemistry, 38(8), 2503-2507.

- Benavides Ballesteros, H. O., \& León Aristizabal, G. E. (2007). Información técnica sobre gases de efecto invernadero y cambio climático (Nota técnica de IDEAM. IDEAMMETEO/008-2007). Recuperado e 2 de febrero de 2015, de http://www.ideam.gov.co/documents/21021/21138/ Gases+de+Efecto+Invernadero+y+el+Cambio+Climatico. pdf/7fabbbd2-9300-4280-befe-cl 1 cf15f06dd

- Burkhardt III, J. J., Heath, G. A., \& Turchi, C. S. (2011). Life cycle assessment of a parabolic trough concentrating solar power plant and the impacts of key design alternatives. Environmental Science \& Technology, 45(6), 2457-2464. Recuperado el 9 de octubre de 2015, de http://pubs.acs.org/doi/pdf/10.1021/ es 1033266

- Burney, J., Woltering, L., Burke, M., Naylor, R., \& Pasternak, D. (2010). Solar-powedered drip irrigation enhances food security in the Sudano-Sahel. Proceeding of the National Academy of Sciences of United States of America, 107(5), 1848-1853.

- Deluchi, M., \& Jacobson, M. Z. (2011). Providing all global energy with wind, water, and solar power, Part II: Reliability, system and transmission costs, and policies. Energy Policy, 39(3), $1170-1190$.

- Dubey, S., Jadhav, N., \& Zahirova, B. (2013). Socio-Economic and Environmental Impacts of Silicon Based Photovoltaic (PV) Technologies. Energy Procedia, 33, 322-334. Recuperado el 
25 de septiembre de 2015, de http://www.sciencedirect. com.consultaremota.upb.edu.co/science/article/pii/ S1876610213000830

- Fthenakis, V., \& Chul Kim, H. (2009). Land use and electricity generation: A life-cycle analysis. Renewable and Sustainable Energy Reviews, 13(6-7), 1465-1474.

- Fthenakis, V., Moskowitz, P., \& Lee, J. (1984). Manufacture of amorphous silicon and GaAs thin film solar cells: an identification of potential health and safety hazards Solar Cells. Biomedical and Environmental Assesment Division, 13(1), 43-58.

- Gagnon, L., Bélanger, C., \& Uchiyama, Y. (2002). Life-cycle assessment of electricity generation options: The status of research in year 2001. Energy Policy, 30(14), 1267-1278.

- Grigoleit, T., \& Lenkeit, D. (2012). The renewable energy industry in Germany. A glance at industry promotion policies in selected energy sectors. Energia Ambiente e Innovazione, 3. Recuperado el 10 de marzo de 2015, de http://www.gtai. de/GTAI/Content/EN/Invest/_SharedDocs/Downloads/GTAI/ Articles/The-renewable-energy-industry-in-germany.pdf

- Gutiérrez, C. (2001). Small hydro: Policy and potential in Spain. Renewable Energy World, 4(5), 148-152.

- Hernandez, R., Easter, S., Murphy Mariscal, M., Maestre, F., Tavassoli, M., Allen, E., ... Michael, A. (2014). Environmental impacts of utility-scale solar energy. Renewable and Sustainable Energy Reviews, 29, 766-779.

- Herrán, C. (2012). El cambio climático y sus consecuencias para América Latina. México: Proyecto Energía y Clima de la Fundación Friedrich Ebert (FES). Recuperado el 2 de febrero de 2015, de http://library.fes.de/pdf-files/bueros/laenergiayclima/09164.pdf

- Intergovernmental Panel on Climate Change. (2012). Special Report on Renewable Enery Sources and Climate Change Mitigation (SRREN). New York, US: Cambridge University Press.

(2014). Cambio climático 2014: Impactos, adaptación y vulnerabilidad-Resumen para responsables de políticas. Recuperado el 12 de marzo de 2015, de https://www. ipcc.ch/pdf/assessment-report/ar5/wg2/ar5_wgll_spm_es.pdf

- International Energy Agency. (septiembre 2014). Publications. Recuperado el 25 de noviembre de 2015, de http://www.iea. org/publications/freepublications/publication/

- Lauber, V., \& Mez, L. (2004). Three decades of renewable electricity policies in Germany. Energy \& Environment, 15(4), 599-623.

- Marland, G., Boden, T., \& Andres, R. J. (2013). Carbon Dioxide Information Analysis Center CDIAC. Global, Regional, and
National Fossil-Fuel CO2 Emissions. Tennessee, US: Oak Ridge National Laboratory US Department of Energy.

- Martinot, E. (2001). World bank energy projects in China: Influences on environmental protection. Energy Policy, 29(8), 581-594.

- National Renewable Energy Laboratory. (2004). Renewable Energy in China. Golden, CO: NREL. Recuperado el 15 de marzo de 2015, de www.nrel.gov.

- Naturalgas.org. (2013). Natural Gas and the Environment. Recuperado el 15 de octubre de 2015, de http://naturalgas. org/environment/naturalgas/

- Nelson, J., Ghambir, A., \& Ekins-Daukes, N. (2014). Solar power for CO2 mitigation (Briefing paper 11). Imperial College. London, UK: Grantham Institute for Climate Change.

- Office of Indian Energy and Economic Development. (2015). Tribal Energy and Environmental Information Clearinghouse. Recuperado el 25 de noviembre de 2015, de http://teeic. indianaffairs.gov/aboutus/index.htm

- Pepper, I., Gerba, C., \& Newby, D. (2009). Soil: a public health threat or savior? Critical Reviews in Environmental Science and Technology, 39(5), 416-432.

- Ravi, S., D'Odorico, P., Brashear, D., Field, J., Goudie, A., Huxman, T., ... Zobeck, T. (2011). Aeolian processes and the biosphere. Review of Geophysics, 49(RG3001), 1-45.

- Rodríguez-Sierra, M. L., \& Serrano-Guzmán, M. F. (2015). Emisiones del carbón por diferentes tecnologías, proyectadas mediante el software LEAP al año 2050 [Entregable del Semillero de Prospectiva Energética Colombia 2050]. Bucaramanga, Santander, Colombia: ECOPETROL, S. A.-UIS-UNAB-UPB.

- Sahu, B. K. (2015). A Study on global solar PV energy developments and policies with special focus on the top ten solar PV power producing countries. Renewable and Sustainable Energy Reviews, 43, 621-634.

- Sinha, P., Kriegner, C., Schew, W., Kaczmar, S., Traister, M., \& Wilson, D. (2008). Regulatory policy governing cadmiumtelluride photovoltaics: A case study contrasting life cycle management with the precautionary principle. Energy Policy, $36(1), 381-387$

- Sullivan, J. L., \& Gaines, L. (2012). Status of life cycle inventories for batteries. Energy Conversion and Management, 134-148. doi: 10.1016/j.enconman.2012.01.001

- Torresol Energy Investments (2010 a). Torresol Energy reinventing solar power. Tecnología de captadores cilindro parabólicos. Recuperado el 7 de octubre de 2015, de http://www. torresolenergy.com/TORRESOL/tecnologia-colectores-cilindroparabolicos/es 


\section{IIVESTIGAGIÓn Y CUERGIA DE \\ LA UחIVERSIDAD AUTÓNOMA \\ DE RGUASCALIERTES}

$\bullet$

(2010 b). Torresol Energy reinventing solar power. Tecnología de torre central. Recuperado el 8 de octubre de 2015, de http://www.torresolenergy.com/TORRESOL/ tecnologia-torre-central/es

- Trieb, F., Schillings, C., Pregger, T., \& O'Sullivan, M. (2012). Solarelectricity imports from the Middle East and North Africa to Europe. Energy Policy, 42 (C), 341-353.

- Tsoutsos, T., Frantzeskaki, N., \& Gekas, V. (2005). Environmental impacts from the solar energy technologies. Energy Policy, 33(3), 289-296. Recuperado el 25 de septiembre de 2015, de http://www.sciencedirect.com.consultaremota.upb.edu.co/ science/article/pii/S0301421503002416
- Unidad de Planeación Minero-Energética. (2015). Plan Energético Nacional Colombia: Ideario Energético 2050. Bogotá, Colombia: Autor.

- US Department of Energy. (2008). Solar power could provide $10 \%$ of U.S. electricity by 2025 . Mother Earth News. The Original Guide to Live Wisely, 25 de junio de 2008 [Reimpresión de EERE Network News, hoja informativa del Departamento de Energía de EE. UU.] Recuperado de http://www.motherearthnews. com/renewable-energy/solar-power/solar-power-potential

- Yanrui, W. (2003). Deregulation and growth in China's energy sector: A review of recent development. Energy Policy, 31 (13), 1417-1425. 